\section{DETECTION OF PUMP FAULTS BASED ON SOUND SIGNALS USING NON-NEGATIVE MATRIX FACTORIZATION}

Anindita Adikaputri Vinayaa*, Fitri Nurmaulidaha, Dhany

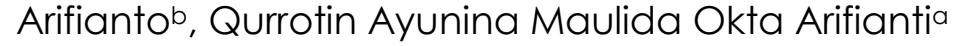

aDepartment of Engineering Management, Universitas Internasional Semen Indonesia, Gresik, East Java, Indonesia bDepartment of Engineering Physics, Institut Teknologi Sepuluh Nopember, Surabaya, East Java, Indonesia
Article history

Received

19 October 2018

Received in revised form

29 October 2019

Accepted

16 January 2020

Published online

27 February 2020

*Corresponding author anindita.vinaya@visi.ac.id

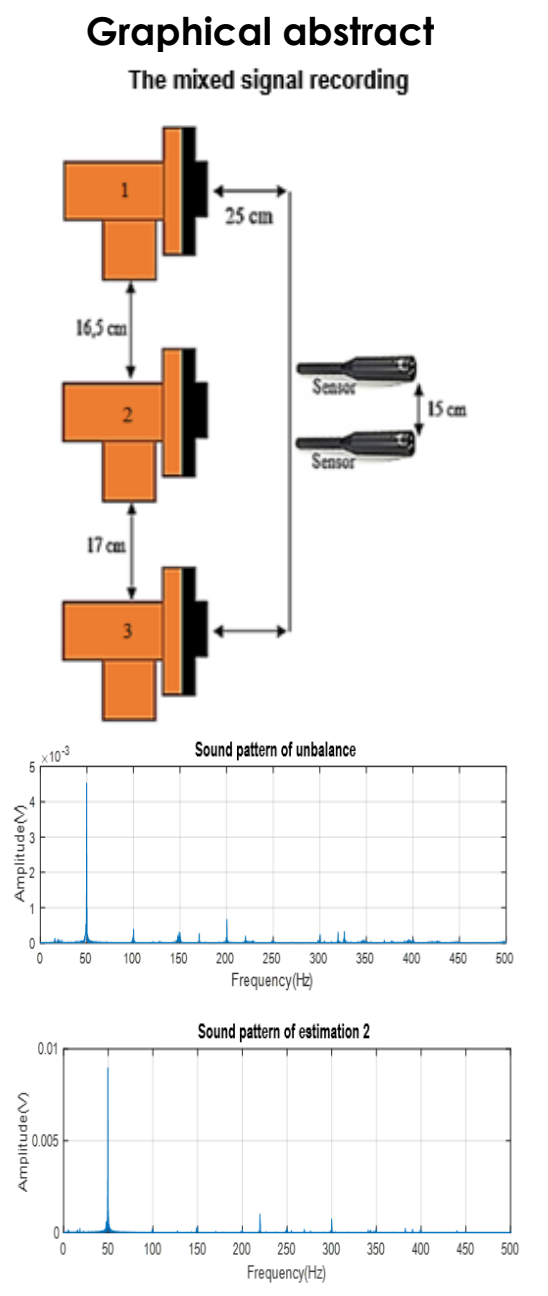

\begin{abstract}
Maintenance is very closely related to the performance of the production process. An alternative method that can be used to determine the damage to the engine is from the analysis of the sound pattern produced. If the sound source is more than one, then there will be signal mixing, and it will be a challenge in detecting damage to the engine. In this study, mixed signals will be separated. Separation of mixed sound signals was done using non-negative matrix factorization (NMF) method. Overall this study is aimed at detecting unbalance, misalignment, and bearing faults at pumps with microphones as sensors. The pumps used in this study were three pumps, where each pump had different conditions (unbalance, misalignment, and bearing fault). All three pumps have $3000 \mathrm{rpm}$. In this study, the recording process was carried out for $5 \mathrm{~s}$. In this study, we also compare the location of the instantaneous frequency in full spectrum and corresponding frequency in local spectrum, and the distance between the spectra via the log spectral distance from the baseline signal and the estimated signal. Based on the instantaneous frequency approach, no error was found because of the instantaneous frequency suitability of the unbalanced machine condition with the estimated signal in the mixing configuration of three sources with two sensors. From the log spectral instance (LSD) results, the smallest value was obtained the smallest value in estimation 2 , which tends to approach the unbalance condition with the LSD value of 1.0889 . The most significant relative error is the estimated misalignment signal with a value of 11.2. However, overall damage can still be identified based on the pattern formed and some statistical parameters.
\end{abstract}

Keywords: Sound pattern, non-negative matrix factorization, pump faults, instantaneous frequency, log spectral distance 


\subsection{INTRODUCTION}

Maintenance is essential to ensure that a machine has the availability or high availability, so that it can carry out its functions in the production process. One of the methods used in industrial maintenance is a condition based monitoring/predictive maintenance [1]-[2]. Gelle et al. in 2000 stated that most of the energy of a vibration is passed on through the emission of sound. Based on the results of his research, sensors that receive acoustic information can become a substitute for sensor vibration to detect damage a machine in noncontact [2]. However, the technique of condition monitoring-based acoustic emission is susceptible to noise emanating from the source machine to the other. When there is only one acoustic signal generated, then the signal will easily be processed to identify damage.If there is more than one acoustic signal generated, the separation of mixed signals will be necessary. Separation is used to extract the signal source from other signals [2]-[3].

Separation of mixed signals is a way of reconstructing the signal source that had been mixed. Blind source separation (BSS) is a form of unsupervised learning method,to extract hidden variables from a given datum. Furthermore, the hidden variable can be used for modeling needs.

Many methods are used to separate the mixed signals, such as full rank [3], parallel factor [4], independent component analysis (ICA) of both the mixed sound signals [5]-[6], images [7], and others [8].

The process of linear instantaneous or convolutive mixing will also affect the result of the separation. Research regarding separation of mixed signals from the engine has been carried out with various methods of research. Gelle et al. used an algorithm to separate mixed signal demixing vibration and the acoustic mix of signals from two machines that are coupled [2]. Through simulation, the bilinear form by blending approach to the superposition was used by Ypma et al. to separate the signals that are mixed [9].

The ICA method has been used to separate mixed signals on the pump in an anechoic chamber by linear instantaneous mixing [5] and in open spaces with convolutive mixing [10]. The multiway array decomposition [11] method has also been used to obtain estimation signals from 4 machines in pump machines [12]. In 2015, the same method was used to separate the signal on the pump engine where the convolution mixing process was used [13]. Based on the results of his research, the source signal can be reconstructed, but the problem of ambiguity and localization of the source signal has been negligible.

In this study, we proposed the concept of minimizing ambiguity by using a non-negative matrix factorization (NMF) approach. Separation of the mixed signal was carried out through BSS using NMF by decomposing the matrix that assumes that the source signal and the mixing signal are non-negative. This approach is the development of advanced blind signal separation in terms of feature extraction, clustering algorithms and performance evaluation based on initial knowledge and morphology of the data [14]-[15] Through this approach, each constituent component of the estimated data actually has meaning and does not produce new noise that can cause interpretation errors [15]. Based on the simulation results, the concept can properly separate the signal's speech and music [14][15]. The separation of the acoustic signal from the engine is a daunting task, because of its limited alphabet and variations in intonation compared with signal speech [16].

\subsection{METHODOLOGY}

A mixed $Z$ signal can be produced through superposition and convolution mixing of source signals $Y_{1}, Y_{2}$ and $Y_{i}$ with $X$ mixing matrix [17]. Equations 1 and 2 are the modeling of linear instantaneous mixing.

$$
Z(t)=\sum_{b=1}^{B} X . Y(t)+n(t)
$$

where

$X=$ mixing coefficient matrix size $A \times B$ (number of sensors $x$ number of sources)

$Y=\left[Y_{1}, Y_{2}, \ldots . Y_{1}\right]^{\top}, B \times 1$ matrix column vector (collection of sources)

$n=$ noise

$Z=\left[\begin{array}{llll}Z_{1}, Z_{2}, \ldots & Z_{1}\end{array}\right]^{\top}$ represents a mixed signal size $A \times 1$ matrix as shown in Figure 1.

In BSS, mixed signals are important information that will be used to reconstruct the sound sources. The mixed signals can be represented by a set of random value as shown in Figure 1. Several processes will be used to cluster the mixture.



Figure 1 Example of mixed-signal representation

Matrix $W$ as the basis matrix and matrix $V$ as an activation matrix will be estimated by maximizing the likelihood to be able to estimate the source signal $Y$. The $Y(n, f)$ matrix is the matrix that will be broken down into $W_{b}$ and $V_{b}$ matrices in which each component has $a$ 
non-negative value, expressed by the following equation:

$$
|Y(n, f)|^{2} \approx W_{b} V_{b}
$$

To be able to obtain the source signal, generally, the mixing matrix and separator matrix must be obtained first. Underdetermined conditions (the number of sensors is less than the number of sources), usually requires a more complex procedure to estimate the initial information from the source signal. Permutation approach will be used to assist in estimating source signals that can trigger uncertainty. In general, the data/signal obtained is partially non-negative or nonnegative, and the signal has a sparse representation. For such data, it would be better to establish a constraint to extract non-negative and sparse components to avoid ambiguity and unpredictable results. Classical approaches cannot guarantee the optimization of non-negativity. NMF helps to solve problems through data representation of each component with non-negativity and spatial constraint functions [14]-[15]. A zero number in the NMF means absence, whereas a positive number indicates the presence of a component. In the NMF model, factors $W$ and $V$ are assumed to be non-negative components.

Unlike the ICA approach, the assumption of independence in signals does not need to be used on NMF. In this research, Itakura-Saito divergence was used to estimate the likelihood of $W$ and $V$, which is equivalent to the NMF of the Y spectra function. [18].

$$
\begin{aligned}
& -\log p\left(Y_{b} \mid W_{b} V_{b}\right) \\
& =\sum_{n, f} d_{I S}\left(|y(n, f)|^{2} \mid W_{b}(n, f) V_{b}(n, f)\right)
\end{aligned}
$$

where, $d_{I S}(g \mid h)=\frac{g}{h}-\log \left(\frac{g}{h}\right)-1, \quad d_{I S}$ is Itakura-Saito divergence. In addition to the Itakura Saito divergence criteria, Kullback-Leibler criteria and Euclidean distance can also be used.

Ensemble data can be modeled and interpreted in a non-negative way and sparse representation. This approach is the development of BSS based on the initial knowledge of the data and the function of morphological data criteria. Given the sparsity and non-negativity limitations, a variance can decrease. In this study, noise is assumed by the equation: $\sum_{n, n f}=$ $\sigma_{n}{ }^{2}$.I, where I is the identity matrix and $\sigma_{n}{ }^{2}$ is the small noise variance. The maximum likelihood estimate is done by minimizing:

$$
C(\theta)=\sum_{n f} \operatorname{trace}\left(\left[Z_{n f} . Z_{n f}^{H}\right] \sum_{Z, n f}^{-1} \theta\right)+\log \operatorname{det} \sum_{z, n f} \theta
$$

The above criteria are still full of uncertainty of scale, phase, and permutation. In phase and scale, $\theta$ which is a combination of components $X, W$ and $V$ can be stated as:

$$
\theta=\left\{\left(X_{f} D_{f}^{-1}\right)_{f}\left(\operatorname{diag}\left(\left[\left|d_{b b, f}\right|^{2}\right]_{f}\right) W_{b} K_{b}^{-1}\right)_{b}\left(K_{b} \cdot V_{b}\right)_{b}\right\}
$$

where $\left(D_{f}\right)_{f}$ and $\left(K_{b}\right)_{b}$ are complex and non-negative sets of diagonal matrices. Scale and phase ambiguity can be eliminated by changing $\sum_{a}\left|x_{a b, f}\right|^{2}=1$ (scaling rows of $W_{b}$ ) and $\sum_{f} W_{f k}=1$ (scaling rows of $V_{b}$ ) [18]. The above criteria were then continuously titrated to maximize the likelihood. The likelihood maximization algorithm for all data can use expectation maximization algorithms.

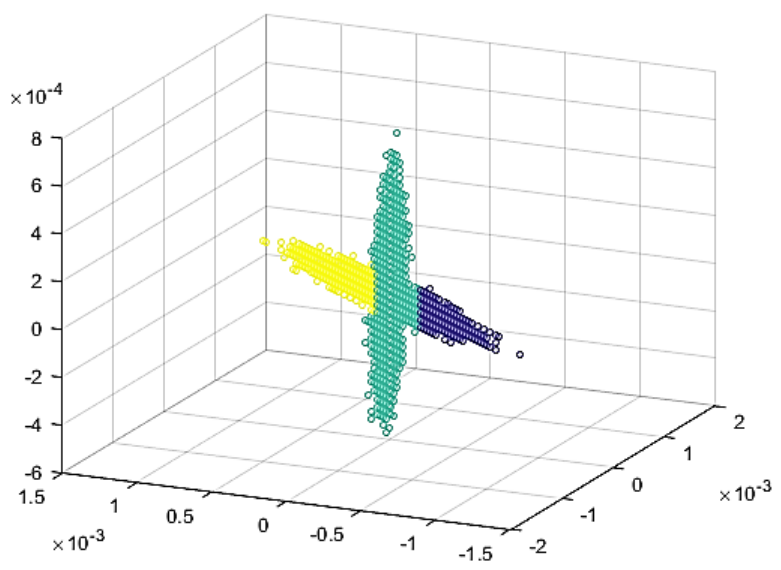

Figure 2 Results of the likelihood maximization

Figure 2 shows the representation of the signal after the estimation and likelihood maximization steps. The signals are grouped into three, according to the sound source used. Based on the results of the likelihood maximization, the equation $Y \approx W \vee$ must be met to reconstruct the signal. Using Wiener filtering criteria, the reconstruction signal will be obtained. Wiener filtering was used in this study because the engine sound signal is a stochastic (random) signal. The Wiener filter involves a linear estimate of the desired signal sequence from other related signals by using the minimum mean square error criterion. The presence of a Wiener filter can affect how close the resemblance or relationship of the original signal to the estimated signal [18].

Furthermore, the reconstruction signal in the timefrequency domain will be returned to the time domain with the inverse short time Fourier transform. In order to determine the condition of the machine, the signal in the time domain is transformed to the frequency domain. Representations in the frequency spectrum are made to make it easier in identifying the engine damage.

Machine sound data retrieval in this research was carried out in two phases, i.e., baseline signal retrieval (one source and one sensor) and signal retrieval from a mixed sound source (multichannel) using omnidirectional microphone type. The baseline signal was used as a comparison with the signal resulting from the separation. The recording process was carried out in a semi-anechoic room. 
The object to be studied was a pumping plant. The pumps used in this study were three pumps, where each pump had different conditions (unbalance, misalignment, and bearing fault). Unbalance is a condition where the center of mass of the shaft is not located at the center of mass of the rotation. Unbalance damage is created by adding a weight of $8 \mathrm{~g}$ of mixed iron to the impeller, misalignment is created by adding coupling and shaft weighing $129 \mathrm{~g}$ at the pump. Coupling and shaft are mutually mounted not straight. Bearing damage is created by hitting on the outer bearing with a hammer. All three pumps have $3000 \mathrm{rpm}$ and have similar specifications:

$\begin{array}{ll}\text { Maximum capacity } & : 30 \mathrm{~L} / \mathrm{m} \\ \text { Capacity at } 12 \mathrm{~m} & : 18 \mathrm{~L} / \mathrm{m} \\ \text { Power suction } & : 9 \mathrm{~m} \\ \text { Maximum total head } & : 30 \mathrm{~m} \\ \text { Motor power } & : 125 \mathrm{~W} \\ \text { Suction pipe } & : 1 \mathrm{inch}(25 \mathrm{~mm}) \\ \text { Push pipe } & : 1 \mathrm{inch}(25 \mathrm{~mm}) \\ \text { Weight } & : 5.4 \mathrm{~kg}\end{array}$

The pump has ball bearings, with the following specifications:

$\begin{array}{ll}\text { Outside diameter } & : 26 \mathrm{~mm} \\ \text { Inside diameter } & : 9 \mathrm{~mm} \\ \text { Pitch diameter } & : 19.6 \mathrm{~mm} \\ \text { Roller diameter } & : 4.2 \mathrm{~mm} \\ \text { Outer ring width } & : 8 \mathrm{~mm} \\ \text { Number of balls } & : 7\end{array}$

In this study the sensor used is dbx RTA-condenser microphone with an omni-directional type polar pattern. Frequency range of microphone is $20-20.000 \mathrm{~Hz}$ and sensor sensitivity is $-63 \mathrm{~dB} \pm 3 \mathrm{~dB}$. The USB audio interface used is Focusrite $18 \mathrm{i} 20$ with a sampling frequency of $48 \mathrm{kHz}$. The software used in recording is Adobe Audition 2015. In this study, the recording process was carried out for $5 \mathrm{~s}$ and saved with the .wav format. In data retrieval, the baseline record between the source of the sound and the source was $5 \mathrm{~cm}$ (Figure 3). The recording data were collected at each pump.

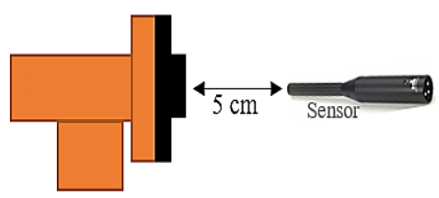

Figure 3 The configuration of baseline data collection

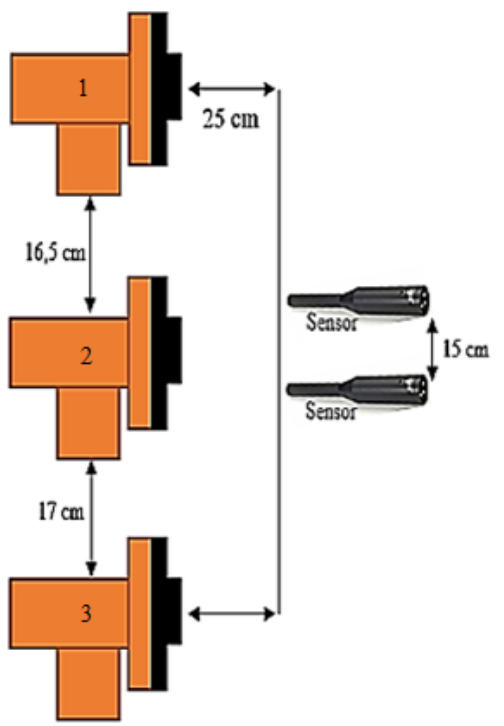

Figure 4 The configuration of mixed data collection

In mixed configuration settings, two sensors are used to record three sources. The configuration used in recording mixed signals can be seen in Figure 4, where pump 1 is a pump with unbalance condition, pump 2 is a pump with misalignment condition, and pump 3 is a pump with bearing fault. The number of estimated signals produced is parallel to the number of sources used.

We use the log spectral distance (LSD) to test the performance of the proposed approach in separating mixed signals when superposition mixing was carried out. The mixed signal that had been obtained from the recording results were separated by decomposing a matrix into several matrices in which components are non-negative values with the NMF. The sampling frequency is reduced to $16.000 \mathrm{~Hz}$ to speed up the data processing. To be able to determine the condition of the engine, the reconstruction signal in the time domain was transformed to the frequency domain with Fast Fourier transform [12]. Representations in the frequency spectrum were carried out to make it easier in detecting pump faults.

There are several parameters that can be used to detect machine conditions through signals in the time domain [24]. Some of them are kurtosis (K), crest factor (CF), and shape factor(SF).

$$
\begin{gathered}
K=\frac{\frac{1}{N} \sum_{i=1}^{N}\left(Y_{i}-\mu\right)^{4}}{\sigma^{4}} \\
C F=\frac{Y_{\max }-Y_{\min }}{2 . Y_{r m s}} \\
S F=\frac{Y_{r m s}}{\frac{1}{N} \sum_{i=1}^{N}\left|Y_{i}\right|}
\end{gathered}
$$


where $N$ is the number of samples, $\mu$ is the average value, $\sigma$ is the standard deviation, $Y_{\max }$ is the maximum value of data $(Y), Y_{\min }$ is the minimum value of data, $Y_{r m s}$ is the root mean square of data.

\subsection{RESULTS AND DISCUSSION}

Figure 5 shows that the baseline signal between engine conditions is not the same. Meaning, each engine condition has different characteristics. The frequency of the machine could not be seen because it was still in the time domain. In order to get the frequency spectrum of the baseline signal, it transformed data from the time domain into the frequency domain.
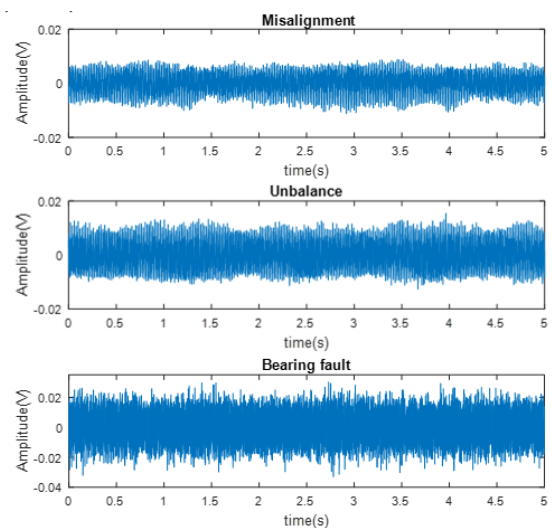

Figure 5 Baseline signal from pumps in time domain

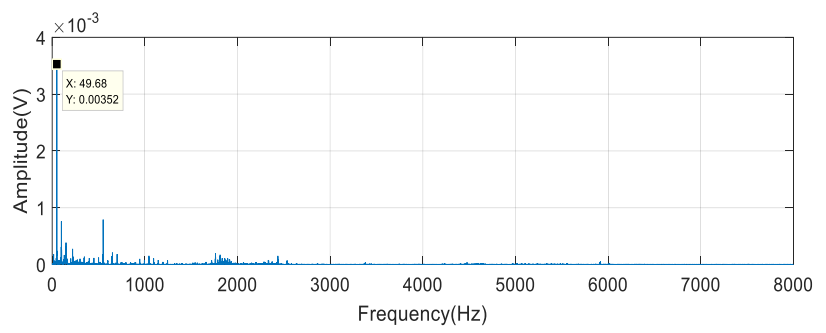

(a)

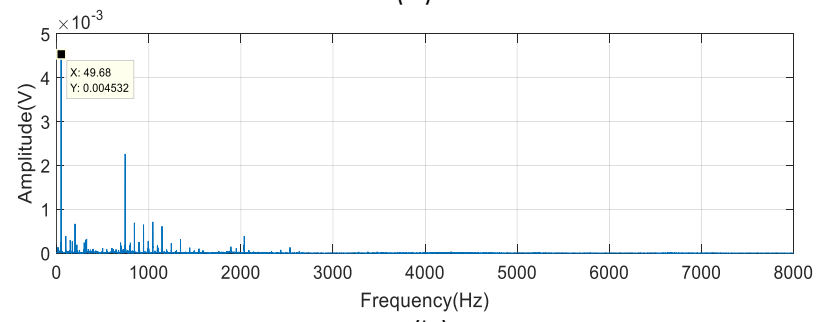

(b)

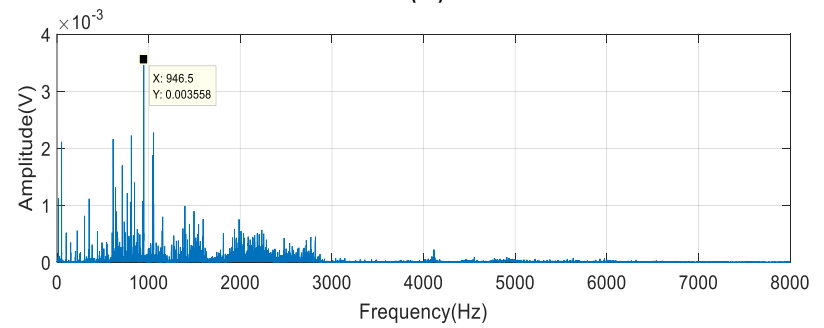

(c)

Figure 6 Baseline signal from pumps in frequency domain: (a) misalignment, (b) unbalance, and (c) bearing fault
In Figures $6 \mathrm{a}$ and $6 \mathrm{~b}$, the instantaneous frequencies of the pump with misalignment condition and unbalance condition were $49.68 \mathrm{~Hz}$. The instantaneous frequency can be seen from the highest peak value. In this study, the pump used is a $3000 \mathrm{rpm}$ rotation pump. Hence, the value of $1 \times$ the fundamental frequency at this pump is $50 \mathrm{~Hz}$. In Figures $6 \mathrm{a}$ and $6 \mathrm{~b}$, the instantaneous frequency is almost the same as $1 \times$ the fundamental frequency. Misalignment is indicated by a high amplitude that appears on $1 \times$ of the fundamental frequency, $2 x$ the fundamental frequency and so on, whereas in unbalance, high amplitude only appears in $1 x$ the fundamental frequency. [19]- [20] The instantaneous frequency value of the pump with a bearing fault condition is $946.5 \mathrm{~Hz}$. In such condition, there were some high amplitudes and ripple at high frequency (Figure $5 c)$. This is because damaged bearings or bearings can cause the shaft to rotate with the buffer, not in the correct position, which results in much vibration at the pump. [21]- [22] Spectrum differences from each machine will be more easily analyzed in the local spectrum as shown in Figure 7. The frequency range used is 0 to $500 \mathrm{~Hz}$. The conditions of misalignment and unbalance are seen more clearly in local spectrum representations. Misalignment is indicated by high amplitude at $1 \times \mathrm{rpm}, 2 \times \mathrm{rpm}$, and $3 \times \mathrm{rpm}$. The frequencies of $49.68 \mathrm{~Hz}, 99.3 \mathrm{~Hz}, 149.2 \mathrm{~Hz}$ approach $1 \times$ rpm, $2 \times$ rpm, and $3 \times$ rpm. The high amplitude at $1 \times$ rpm indicates unbalance conditions. Misalignment, unbalance, and bearing fault indications are as stated previously.

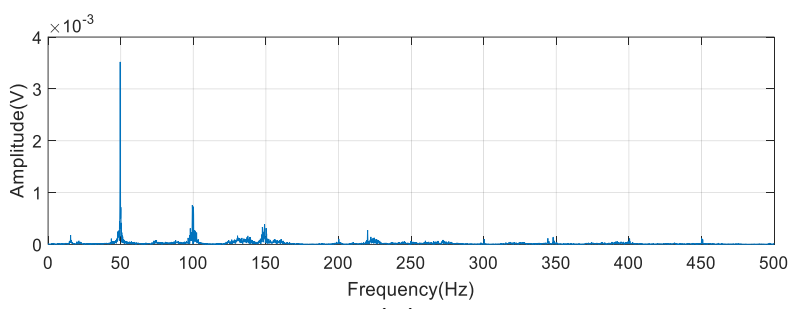

(a)

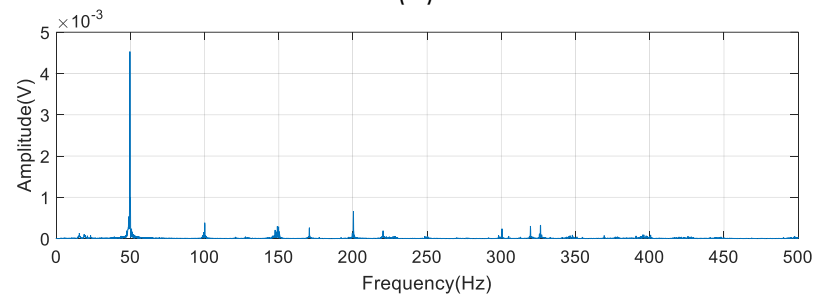

(b)

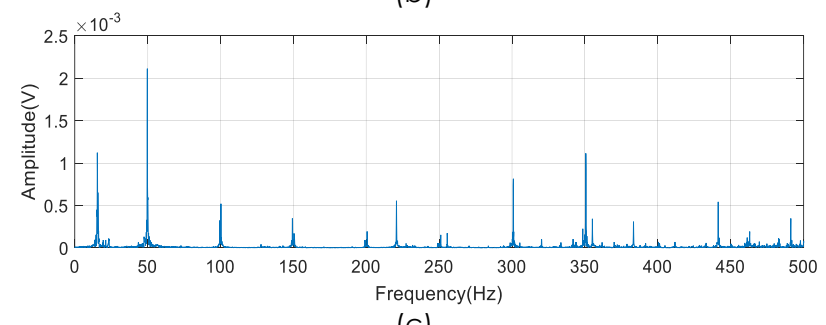

(c)

Figure 7 Baseline signals in the local spectrum: (a) misalignment, (b) unbalance, and (c) bearing fault 
Mixed signals that had been obtained according to the experimental setup in Figure 4 were separated. The mixed signals were separated by using the NMF algorithm with the mixing process used an instantaneous linear mixture. After advancing through several stages of the NMF algorithm, estimated signals were generated. The estimated signals were then plotted in the full spectrum and the local spectrum as shown in Figure 8 and Figure 9, respectively.
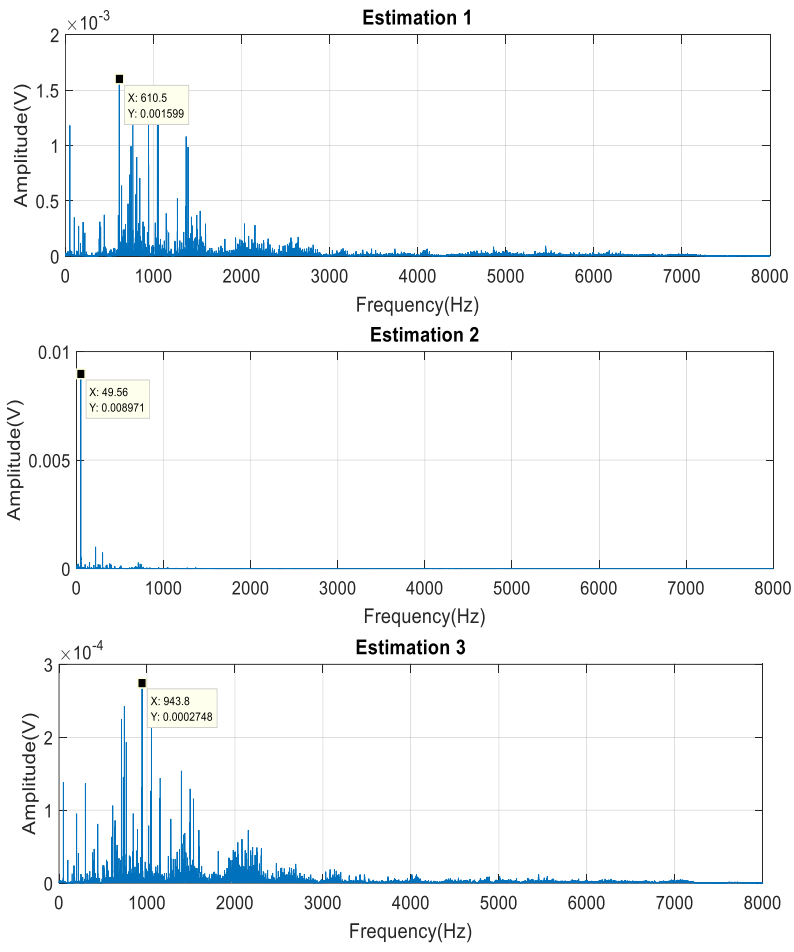

Figure 8 Estimated signals in the full spectrum

In the local spectrum representation, estimation 1 approaches the misalignment condition marked by the appearance of amplitude at $1 \times \mathrm{rpm}$ and $2 \times \mathrm{rpm}$. Estimation 2 is similar to the unbalance condition both in full spectrum and local spectrum representation.
Based on the sound pattern formed, estimation 3 indicates bearing damage in the presence of harmonic frequencies. Corresponding frequency location of the baseline signal and estimation in the local spectrum are shown in Table 1.
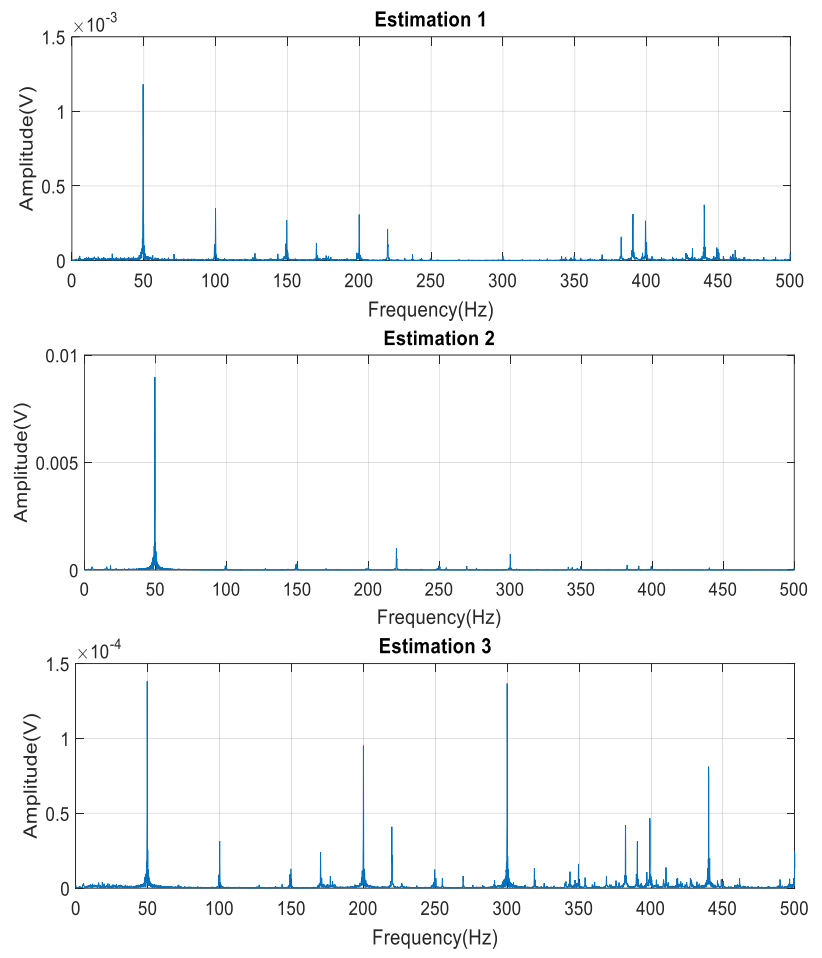

Figure 9 Estimated signals in the local spectrum

These different spectrum patterns illustrate the characteristics of the machine. The next step was to compare the estimated signal of the result of separation with the baseline signal. Comparison of the estimated signal spectrum and the baseline signal is shown in Figures 10. In Figure 10, one of the estimation signals is plotted with the baseline signal, so that the differences between the estimation signal and the baseline signal can be easily identified.

Table 1 Frequency related to pump faults in local spectrum

\begin{tabular}{|c|c|c|c|c|c|c|c|c|c|}
\hline \multicolumn{2}{|c|}{ Signals } & \multicolumn{8}{|c|}{ Corresponding Frequency $(\mathrm{Hz})$} \\
\hline & & $1 \times$ & $2 x$ & $3 x$ & $4 \times$ & $5 x$ & $6 x$ & $7 x$ & $8 x$ \\
\hline \multirow{3}{*}{ Baseline } & Misalignment & 49.68 & 99.3 & 149.2 & & & & & \\
\hline & Unbalance & 49.68 & & & & & & & \\
\hline & Bearing fault & 49.8 & 100.3 & 149.5 & & & 300.9 & 350.6 & \\
\hline \multirow{3}{*}{ Separation result } & Estimation 1 & 49.56 & 99.9 & 149.5 & 200 & & & & \\
\hline & Estimation 2 & 49.56 & & & & & & & \\
\hline & Estimation 3 & 49.56 & 99.9 & & 200 & & 299.9 & 349.5 & 399.2 \\
\hline
\end{tabular}




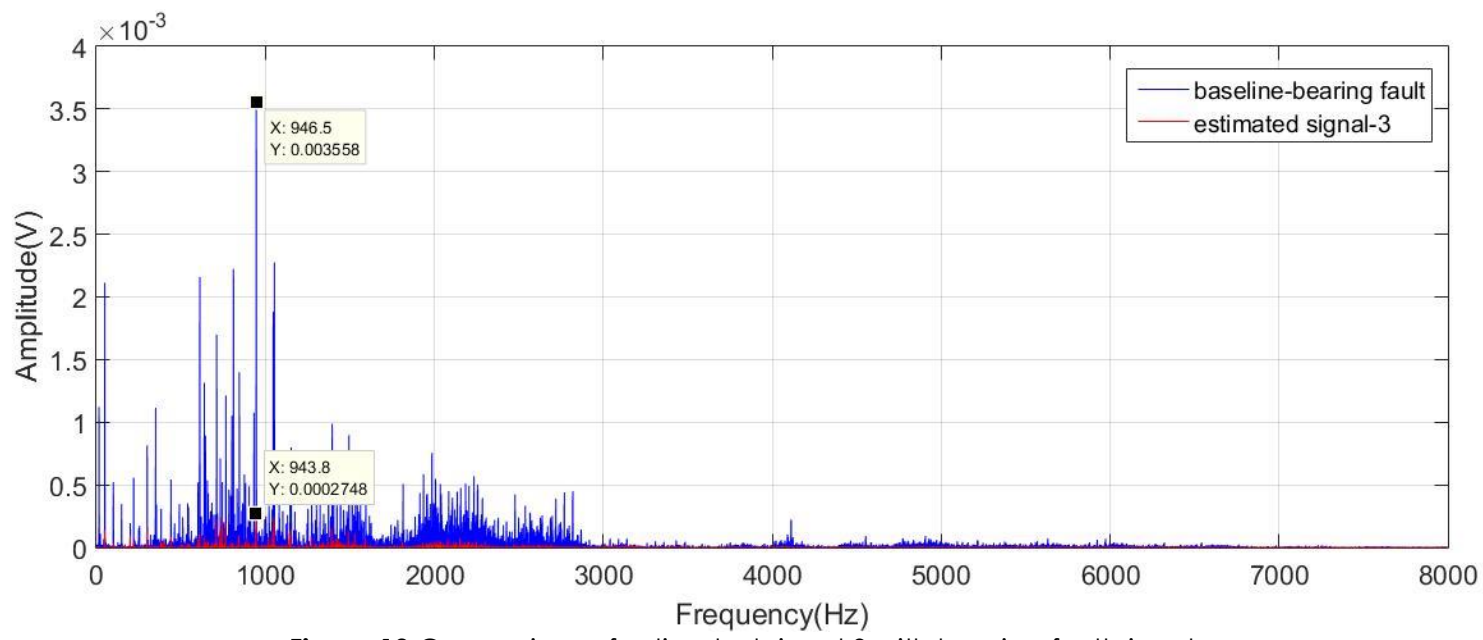

Figure 10 Comparison of estimated signal 3 with bearing fault signal

Based on Figure 10, the instantaneous frequency of the baseline signal is higher than the estimated signal frequency. The instantaneous frequency baseline signal is $946.5 \mathrm{~Hz}$ and appears at an amplitude of $2.97 \times 10^{-3} \mathrm{~V}$, while the estimated instantaneous frequency signal is $943.8 \mathrm{~Hz}$ and appears at an amplitude of $2.476 \times 10^{-4} \mathrm{~V}$. The instantaneous frequency can be seen in Table 2. The relative error of the instantaneous frequency of the estimated signal comparison with the baseline misalignment condition, which has the highest value compared with the others, is 11.2. Having compared the estimated signal 3 with the baseline unbalance condition, no error was found due to the suitability of the instantaneous frequency.

Table 2 The instantaneous frequency of baseline and estimation signal

\begin{tabular}{llll}
\hline Signals & \multicolumn{3}{l}{ Instantaneous frequency } \\
\hline & Misalignment & $\begin{array}{l}\text { Bearing } \\
\text { fault }\end{array}$ & Unbalance \\
\hline Baseline & $49.68 \mathrm{~Hz}$ & $946.5 \mathrm{~Hz}$ & $49.68 \mathrm{~Hz}$ \\
Estimation & $610.5 \mathrm{~Hz}$ & $943.8 \mathrm{~Hz}$ & $49.68 \mathrm{~Hz}$ \\
$\begin{array}{l}\text { Relative } \\
\text { error }\end{array}$ & $\mathbf{1 1 . 2}$ & $\mathbf{0 . 0 0 2 8}$ & $\mathbf{0}$ \\
\hline
\end{tabular}

To discover the size of the performance in the frequency domain, LSD can be applied. LSD is the squared difference of the logarithm of the spectral envelop the original signal $Y(n, f)$ and the estimated signal $Y(n, f)$ [23]. From the LSD results listed in Table 3, the smallest value was obtained in estimation 2 , which tends to approach the unbalance condition with the LSD value of 1.0889, and the largest value, in estimation 3 , which tends to approach bearing fault with LSD value of 2.4989. However, overall damage can still be identified based on the pattern formed.

Table 3 LSD value

\begin{tabular}{lll}
\hline No & Estimation & LSD \\
\hline 1 & Estimation 1-misalignment & 1.8219 \\
2 & Estimation 2-unbalance & 1.0889 \\
3 & Estimation 3-bearing fault & 2.4989 \\
\hline
\end{tabular}

Different conditions of each pump will also be analyzed through signals in the time domain. Each baseline signal and the estimated signal has different statistical characteristics. These characteristics are seen in kurtosis (K), crest factor (CF) and shape factor (SF) values in Table 4. The unbalance signals, both baseline and estimation, have the lowest K, CF, and SF values than others. The highest K, CF and SF values are found in the bearing fault signal that appears in the baseline signal and the estimated signal.

Table 4 Kurtosis, crest factor and shape factor values

\begin{tabular}{lrrrrrr}
\hline \multirow{2}{*}{ Signal } & \multicolumn{2}{c}{ K } & \multicolumn{2}{c}{ CF } & \multicolumn{2}{c}{ SF } \\
\cline { 2 - 7 } & Baseline & Estimation & Baseline & Estimation & Baseline & Estimation \\
Misalignment & 2.55 & 2.95 & 3.24 & 4.24 & 1.21 & 1.24 \\
Unbalance & 2.42 & 1.65 & 2.88 & 2.00 & 1.19 & 1.12 \\
Bearing Fault & 3.01 & 3.01 & 4.14 & 4.31 & 1.25 & 1.25 \\
\hline
\end{tabular}




\subsection{CONCLUSION}

Based on the research that has been done, it can be concluded that the separation of sound signals using NMF method was successfully carried out. The instantaneous frequency of the estimation signals (estimation 2 and estimation 3) resulting from the separation process has approached the instantaneous frequency of the unbalance signal and bearing fault signal. Based on the instantaneous frequency approach, the most significant relative error is the estimated misalignment signal with a value of 11.2. The performance test results, using LSD, obtained the smallest value in the estimation 2 and the largest value in the estimation 3, where the estimation 2 tends to approach the pump machine with an unbalance condition with LSD 1.0889, and the estimation 3 tends to approach the pump with a bearing fault condition with LSD 2.4989. Different conditions of each pump will also be analyzed in the time domain through the value of kurtosis, crest factor and shape factor.

\section{Acknowledgement}

We would like to extend our gratitude to the Direktorat Riset dan Pengabdian kepada Masyarakat (DRPM), Ditjen Penguatan Riset dan Pengembangan, Kementerian Riset, Teknologi, dan Pendidikan Tinggi (Kemenristekdikti) who have funded this research on a collaborative research scheme (Penelitian Kerjasama Perguruan Tinggi) No. 074 / SP2H / LT / K7 / KM / 2018, between Universitas Internasional Semen Indonesia and Institut Teknologi Sepuluh Nopember.

\section{References}

[1] Heng, R. B. W., and Mohd Jailani Mohd Nor. 1998. Statistical Analysis of Sound and Vibration Signals for Monitoring Rolling Element Bearing Condition. Applied Acoustics. 53: 21 1-226.

[2] Gelle, G., Colas, M., and Delaunay, G. 2000. Blind Sources Separation Applied to Rotating Machines Monitoring by Acoustical and Vibrations Analysis. Mechanical Systems and Signal Processing. 14(3): 427-442.

[3] Duong, N. Q., Vincent, E., \& Gribonval, R. 2010. Underdetermined Reverberant Audio Source Separation Using a Full-rank Spatial Covariance Model. IEEE Transactions on Audio, Speech, and Language Processing. 18(7): 1830-1840.

[4] Vinaya, A. A., and Arifianto, D. 2015. Fault Diagnosis of Rotating Machinery based on Acoustic Emission using PARAFAC-Source Separation. IPTEK Journal of Proceedings Series. 1(1): 183-184.

[5] Atmaja, B. T., and Arifianto, D. 2009. Machinery Fault Diagnosis Using Independent Component Analysis (ICA) and Instantaneous Frequency (IF). 2009 International Conference on Instrumentation, Communications, Information Technology, and Biomedical Engineering (ICICI-BME).

[6] Mechefske, C. K. 1998. Objective Machinery Fault Diagnosis Using Fuzzy Logic. Mechanical Systems and Signal Processing. 12(6): 855-862.
[7] Zhiliang Wang,Jian Gao, Chuanxia Jian,Yu Cen and Xin Chen. 2012. OLED Defect Inspection System Development through Independent Component Analysis. TELKOMNIKA Indonesian Journal of Electrical Engineering. 10(8): 23092319.

[8] Chen, Y., \& Meng, J. 2013. Study on BSS Algorithm Used on Fault Diagnosis of Gearbox. Indonesian Journal of Electrical Engineering and Computer Science. 11 (6): 2942-2947.

[9] Ypma, A., Leshem, A., Duin, R. P. 2002. Blind Separation of Rotating Machine Sources: Bilinear Forms and Convolutive Mixtures. Neurocomputing. 49(1): 349-368.

[10] Yunata, A. S. 2010. Identification of Machine Damage Simultaneously in an Open Space with Independent Component Analysis. Thesis in Institut Teknologi Sepuluh Nopember, Surabaya.

[11] Nion, D., Mokios, K. N., Sidiropoulos, N. D., \& Potamianos, A. 2010. Batch and Adaptive PARAFAC-based Blind Separation of Convolutive Speech Mixtures. IEEE Transactions on Audio, Speech, and Language Processing. 18(6): 1193-1207.

[12] Vinaya, A. A., \& Arifianto, D. 2017. Multi-way Array Decomposition on Acoustic Source Separation for Fault Diagnosis of a Motor-pump System. International Journal of Electrical and Computer Engineering (IJECE). 7(6): 3052-3059.

[13] Vinaya, Anindita Adikaputri, and Arifianto, Dhany. 2015. Underdetermined Blind Source Separation based Condition Monitoring. 2015 International Conference on Science in Information Technology (ICSITech IEEE).

[14] Févotte, C., Vincent, E., Ozerov, A. 2018. Audio Source Separation. Single-channel Audio Source Separation with NMF: Divergences, Constraints and Algorithms. Cham. Springer. 1-24.

[15] Cichocki, A., Zdunek, R., Phan, A. H., Amari, S. I. 2009. Nonnegative Matrix and Tensor Factorizations: Applications to Exploratory Multi-way Data Analysis and Blind Source Separation. 1 ed. John Wiley \& Sons.

[16] Knaak, M., Kunter,M., and Filberi, D. 2002. Blind Source Separation for Acoustical Machine Diagnosis. 2002 14th International Conference on Digital Signal Processing Proceedings.

[17] Hyvarinen, O. 2000. Independent Component Analysis: Algorithms and Ahallications. Neural Networks. 13(4-5): 411430.

[18] Ozerov, A., and Févotte, C. 2010. Multichannel Nonnegative Matrix Factorization in Convolutive Mixtures for Audio Source Separation. IEEE Transactions on Audio, Speech, and Language Processing. 18(3): 550-563.

[19] Mobius Institute. Unbalance. [Online]. Available: https://www.mobiusinstitute.com/site2/item.asp?LinkID=800 0\&iVibe $=1 \&$ sTitle=Unbalance. [Accessed 8 October 2018] .

[20] Mobius Institute. Misalignment. [Online]. Available: https://www.mobiusinstitute.com/site2/item.asp?LinkID=100 02\&iVibe $=1$ \&sTitle=Analysis\%20Definitions. [Accessed 8 October 2018].

[21] Mobius Institute. Rolling element bearings. [Online] Available:

https://www.mobiusinstitute.com/site2/item.asp? LinkID=100 03\&iVibe $=1$ \&sTitle=Analysis\%20Definitions. [Accessed 8 October 2018].

[22] Gu, D. S., Choi, B. K. 201 1. Machinery Faults Detection Using Acoustic Emission Signal. Acoustic Waves-From Microdevices to Helioseismology. InTech.

[23] Freudenberger, J., Stenzel, S. \& Venditti, B. 2010. Microphone Diversity Combining for In-car Applications. EURASIP Journal on Advances in Signal Processing. 5.

[24] Van Hecke, B., Qu, Y., \& He, D. 2015. Bearing Fault Diagnosis Based on a New Acoustic Emission Sensor Technique. Proceedings of the Institution of Mechanical Engineers, Part O: Journal of Risk and Reliability. 229(2): 105-118. 